\title{
Non-intubated recovery from refractory cardiogenic shock on percutaneous VA-extracorporeal membrane oxygenation
}

\author{
J. van Houte $\cdot$ D.W. Donker $\cdot$ L.J. Wagenaar $\cdot$ A.P. Slootweg $\cdot$ J.H. Kirkels $\cdot$ D. van Dijk
}

Published online: 5 June 2015

(C) The Author(s) 2015. This article is published with open access at Springerlink.com

\begin{abstract}
We report on the use of percutaneous femoral veno-arterial extracorporeal membrane oxygenation (VAECMO) in a fully awake, non-intubated and spontaneously breathing patient suffering from acute, severe and refractory cardiogenic shock due to a (sub)acute anterior myocardial infarction. Intensified heart failure therapy was closely monitored with a pulmonary artery catheter and allowed gradual weaning off the ECMO support without additional invasive measures, notably without mechanical ventilation. Neurological assessment was possible at all times and complete physical mobilisation was straightforward directly after weaning from ECMO. This limited invasive approach may encourage a more widespread use of percutaneous VA-ECMO.
\end{abstract}

Keywords Veno-arterial extracorporeal membrane oxygenation - Refractory cardiogenic shock · Awake · Non-intubated

D.W. Donker $(\bowtie) \cdot$ J. van Houte $\cdot$ D. van Dijk

Department of Intensive Care Medicine,

University Medical Center Utrecht,

Heidelberglaan 100,

PO Box 85500, 3584 CX Utrecht, The Netherlands

e-mail: D.W.Donker@UMCUtrecht.nl

\section{L.J. Wagenaar}

Department of Cardiology, Thoraxcentrum Twente,

Medisch Spectrum Twente,

Enschede, The Netherlands

\author{
A.P. Slootweg \\ Department of Cardiology, Ziekenhuisgroep Twente, \\ Almelo, The Netherlands
}

J.H. Kirkels

Department of Cardiology, University Medical Center Utrecht,

Utrecht, The Netherlands

\section{Introduction}

Peripheral veno-arterial extracorporeal membrane oxygenation (VA-ECMO) is increasingly used in acute, severe cardiogenic shock refractory to conventional therapy. Although percutaneous femoral insertion of VA-ECMO is straightforward, the procedure is usually performed after intubation under general anaesthesia, and invasive ventilation is continued for the whole period of extracorporeal support.

Here, we show that VA-ECMO can be successfully performed with neither (non-) invasive ventilation nor deep analgosedation.

A 51-year-old man with a history of hypertension and diabetes mellitus presented to the local hospital in cardiogenic shock (blood pressure, $80 / 40 \mathrm{mmHg}$ ). Electrocardiography (ECG) revealed a (sub)acute anterior myocardial infarction complicated by complete atrioventricular block and a slow ventricular escape rhythm of 30 beats per minute (Fig. 1). After initial medical therapy (volume resuscitation, inotropes), the clinical condition allowed urgent transport to an interventional centre. On arrival, immediate transvenous ventricular pacing was commenced, but did not lead to haemodynamic stabilisation. Echocardiography showed preserved wall thickness, but extensive apical and anteroseptal akinesia and inferior hypokinesia, with a large left ventricular (LV) apical thrombus (left ventricular ejection fraction (LVEF), 10\%).

Since cardiogenic shock persisted, VA-ECMO was initiated under local anaesthesia via femoral access $(25 \mathrm{~F}$ venous cannula, 19F arterial cannula) as a bridge to recovery and additional therapy. During the procedure, the patient was fully awake, non-intubated and breathing oxygen through a non-rebreathing facemask. VA-ECMO yielded 4-L/ min flow, and a mean arterial pressure of $60-70 \mathrm{mmHg}$ was achieved. An adequately supported circulation was 


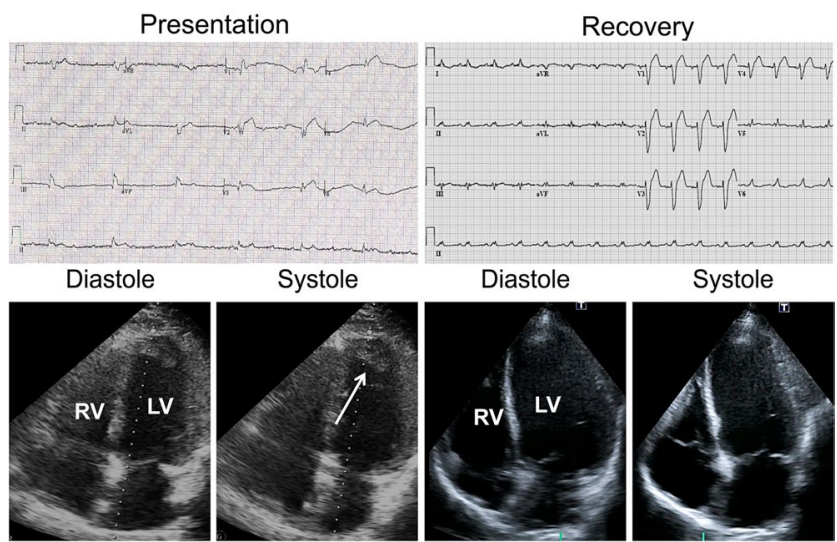

Fig. 1 Serial electrocardiograms (upper panel) and transthoracic echocardiograms (lower panel) on presentation (left) and on recovery 6 weeks later (right) showing a large dilated anterior myocardial infarction with partial recovery of contractile function. Arrow indicates LV apical thrombus, which dissolved over time after therapeutic anticoagulation (recovery). $R V$ right ventricle, $L V$ left ventricle

reflected by normal blood gas analysis, lactate levels, venous oxygen saturation and renal function; sinus rhythm ensued on day 2.

Coronary angiography revealed severe three-vessel disease (proximally occluded right, significantly stenosed circumflex and subtotally occluded left anterior descending coronary artery) unattractive for immediate revascularisation. The clinical course was complicated by obvious signs of pulmonary oedema on serial chest radiographs (Fig. 2) in the absence of dyspnoea (respiratory rate of 15-20 per minute) and with adequate oxygenation $\left(\mathrm{SaO}_{2}, 97-100 \% ; \mathrm{PaO}_{2}\right.$ $>100 \mathrm{mmHg}$ ). Therefore, heart failure therapy (captopril, eplerenone, isosorbide mononitrate) was tailored with a pulmonary artery catheter (PAC) and intensified (furosemide, hydrochlorothiazide) to accomplish negative fluid balances.

After 4 days of VA-ECMO under low-dose continued intravenous analgesia ( $1 \mathrm{mg}$ morphine/h), support flow was gradually reduced $(1 \mathrm{~L} / \mathrm{min}$ per day) under therapeutic heparinisation and PAC monitoring. On day 8 , echocardiography revealed partial LV recovery (LVEF, $25 \%$ ) during normally conducted sinus rhythm (Fig. 1) and $1 \mathrm{~L} / \mathrm{min}$ support flow, allowing weaning off the VA-ECMO.

In a stable condition, the patient was mobilised and discharged from the intensive care unit (ICU) on day 11. Based on myocardial viability studies (mid anteroseptal viability on cardiac magnetic resonance perfusion imaging), a drugeluting stent was placed in the left anterior descending artery on day 21 , followed by clinical discharge on day 37 .

\section{Discussion}

There is accumulating evidence that severe, refractory cardiogenic shock can be supported by percutaneous VA-

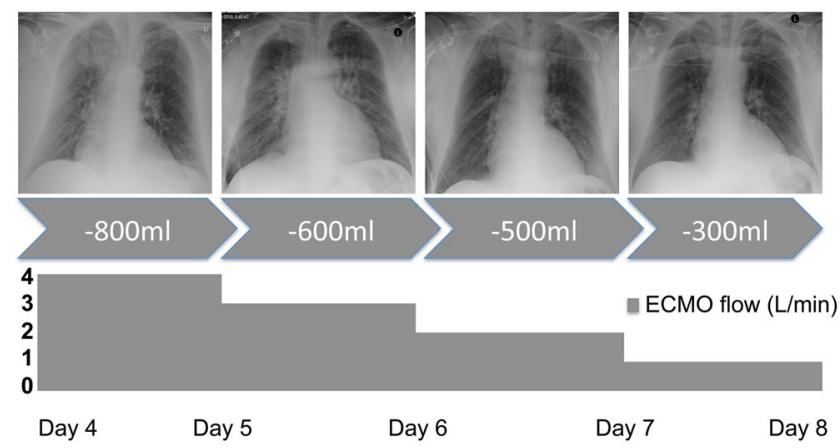

Fig. 2 Serial chest X-rays indicating decrease of pulmonary congestion (upper panel). Daily cumulative fluid balance (middle panel). Daily gradual tapering of ECMO flow $(\mathrm{L} / \mathrm{min})$ during weaning off ECMO support (lower panel)

ECMO as a bridge to recovery, long-term mechanical support and eventually heart transplantation [1].

Yet, VA-ECMO is cumbersome, as patients are generally managed and instrumented in the ICU with invasive ventilation, analgosedation and feeding via a nasogastric tube. This renders VA-ECMO a complex and invasive therapeutic approach with numerous potential ICU-related complications.

In contrast to VA-ECMO standard care, we managed this case without intubation, ventilation or significant analgosedation, which has, to the best of our knowledge, not been reported in this way before [2]. We used PAC monitoring for haemodynamic tailoring and strived for optimal filling pressures (wedge pressures $<15 \mathrm{mmHg}$ ), while allowing adequate systemic VA-ECMO flow. During VA-ECMO weaning, we monitored cardiac recovery by continuously measuring intrinsic cardiac output on gradual daily reduction of support flow (Fig. 1). Moreover, spontaneous respiration was carefully monitored by pulse oximetry and repeated blood gas analyses, while pulmonary oedema was aggressively treated by combined diuretic therapy (Fig. 1). This strategy seems imperative as, for example, pneumonia may render VA-ECMO without intubation impossible [2]. Others have reported on awake VA-ECMO requiring additional percutaneous atrioseptostomy to treat pulmonary congestion, which was not necessary in our patient [3]. Moreover, neurological performance remained unremarkable and could easily be evaluated in this cooperative patient. Neurological assessment is of utmost importance during peripheral VA-ECMO, especially on recovering cardiac function and pending pulmonary oedema, which might cause hypoxaemia of the proximal aortic arch, that is, the Harlequin syndrome.

After roughly a week of VA-ECMO support, cardiac recovery was accompanied by immediate physical mobilisation creating optimal conditions for the subsequent clinical course. 


\section{Conclusion}

VA-ECMO for severe and refractory cardiogenic shock may successfully be performed without mechanical ventilation. Careful monitoring of volume status and respiratory condition is imperative and allows keeping patients comfortable and cooperative also during prolonged periods of extracorporeal support. The limited invasiveness of this stand-alone VA-ECMO approach may encourage a more widespread and accessible use in cardiogenic shock.

Funding None.

Conflict of interest None declared.

Open Access This article is distributed under the terms of the Creative Commons Attribution License which permits any use, distribution, and reproduction in any medium, provided the original author(s) and the source are credited.

\section{References}

1. Nichol G, Karmy-Jones R, Salerno C, et al. Systematic review of percutaneous cardiopulmonary bypass for cardiac arrest or cardiogenic shock states. Resuscitation. 2006;70:381-94.

2. Camboni D, Philipp A, Hirt S, et al. Possibilities and limitations of a miniaturized long-term extracorporeal life support system as bridge to transplantation in a case with biventricular heart failure. Interact Cardiovasc Thorac Surg. 2009;8:168-70.

3. Peter S, Reichelt A, Kaczmarek I, et al. Bridge to heart transplantation in an awake patient by veno-arterial extracorporeal life support and additional left ventricular unloading by atrioseptostomy. Thorac Cardiovasc Surg. 2013. doi:10.1055/s-0032-1332337 